\title{
Introducción
}

En la medida en que se hacen más agudos los problemas de contaminación química, del medioambiente, particularmente en los países industrializados, adquiere mayor importancia el fenómeno de superficie denominado adsorción, concepto con el cual se designa al enriquecimiento de la superficie de adsorbentes sólidos con gases, vapores o líquidos (1).

Este proceso, mediante el cual partículas de sustancias se adhieren a la superficie o incluso poros de una sustancia sólida llamada adsorbente era conocido ya en la antigüedad, pero es en el siglo XVIII cuando se realizan las primeras investigaciones que permiten describir ampliamente el fenomeno. I. Langmuir $(2,3)$ llegó a la conclusión, tras numerosas investigaciones de que las capas adsorbidas tenían un espesor de aproximadamente el tamaño de una molécula. A Langmuir debemos una de las ecuaciones matemáticas más conocidas, para describir el fenómeno de la adsorción.

El desarrrollo de la Química fue planteando nuevos problemas en torno a la adsorción como el estudio de los diversos factores que influyen en ella. Hoy en día gracias a las múltiples investigaciones realizadas, sabemos que la adsorción depende fuertemente de la actividad protónica de la fase liquída $(4,5)$ así como de los grupos oxidos presentes en la superficie del adsorbente (6); de la

Area de Química. Facultad de Ciencias y Humanidades. INTEC. 
superfice de contacto solución-adsorbente; del tamaño de partícula adsorbente; del tipo de poros del adsorbente; de la estructura química del soluto; de la velocidad con que la solución entra en contacto con el adsorbente, de la concentración de la solución, de la presión en el caso de los gases, etc.

\section{Aplicaciones de la adsorción}

La adsorción es el principio en el cual se fundamenta la instalación de filtros industriales, para eliminar partículas de polvo contenidas en el humo de las chimeneas, o sustancias químicas indeseables en líquidos.

Esta técnica encuentra particular uso en el tratamiento del agua potable y de las aguas residuales.

Como técnica de decoloración es muy apreciada, pues una sustancia coloreada puede convertirse en transparente al hacerse pasar por una columna de un adsorbente como por ejemplo el carbón activado. La industria de bebidas alcohólicas recurre permanentemente a la adsorción en la decoloración de rones y licores.

El muy conocido filtro casero en cuyo interior se encuentra un adsorbente como la piedra pómez, o carbón activado basa su acción purificadora en la adsorción.

No puede olvidarse a la industria del papel entre cuyos productos se encuentra el papel de filtro, que retiene las partículas precisamente por adsorción.

La costumbre de muchos criadores de peces de introducir trozos de carbón en las peceras, o las amas de casa de colocar carbón en el refrigerador, son ejemplos de usos populares de la adsorción. En el primer caso, el criador trata de recoger las impurezas orgánicas suspendidas en el agua, adsorbiéndolas en la superficie del carbón. En el segundo caso, se adsorben los olores difundidos en el interior del refrigerador.

Por otra parte las industrias lanzan toneladas de partículas y gases contaminantes anualmente a la atmósfera. Entre los gases más conocidos figuran los óxidos de nitrógeno (NOx) y los de azufre. Particular importancia se asigna al dióxido de azufre $\left(\mathrm{SO}_{2}\right)$ que al reaccionar en la atmósfera con vapor de agua se convierte en gotitas de ácido sulfúrico $\left(\mathrm{H}_{2} \mathrm{SO}_{4}\right)$ que precipitan a la tierra constituyendo la denominada lluvia ácida.

Mediante la instalación de filtros con adsorbentes puede disminuirse sustancialmente la cantidad de emanaciones gaseosas que como el dióxido de azufre o los óxidos de nitrógeno producen el conocido "smog" o la lluvia ácida.

El catalizador que actualmente se exige en varios países desarrollados a los automovilistas para su instalación en los sistemas de escape de los autos, está fundamentado precisamente en la adsorción. 
No puede dejar de citarse a la cromatografía, método de análisis químico de extraordinaria importancia, cuyos fundamentos están en la retención de las sustancias, mediante adsorción en un adsorbente.

Así pues, son múltiples los usos que encuentra la adsorción en diferentes áreas de la vida moderna.

Areas en que se divide el estudio de la adsorción

El estudio de la adsorción suele dividirse en tres grandes áreas, a saber: equilibrio, cinética y dinámica. Estas tres áreas sin embargo guardan una estrecha relación entre sí y a menudo una es auxiliar de la otra.

\section{Equilibrio de adsorción}

Esta área se ocupa del equilibrio entre las fases, líquido-sólido o gas sólido, según el caso. Aquí se encuentra la concentración de la sustancia a ser adsorbida, tanto en la superficie del adsorbente (cantidad adsorbida), como en el seno de la solución (concentración en equilibrio) después que ha transcurrido un período de contacto entre sustancia y adsorbente.

$\mathrm{El}$ equilibrio entre las fases y la medida cuantitativa de adsorción fue descrita por Gibbs y es definida como el exceso de masa (habitualmente en moles) del componente considerado, que corresponde a una unidad de área de la superficie de separación de las fases.

Termodinámica de la adsorción de electrolitos débiles en superficie sólida

La ecuación de adsorción de Gibbs

La entalpia libre $\mathrm{g}$ de un sistema de múltiples componentes es una función de la presión, de la temperatura, de la composición y de la superficie:

$$
g=g(p, T, n i, A)(1)
$$

La diferencial total de esta función es:

$$
\mathrm{dg}=\mathrm{Vdp}-\mathrm{SdT}+\sum_{\mathbf{i}} \mu \mathrm{i} \mathrm{dni}+\sigma \mathrm{dA}
$$

Con la entalpia libre de superficie

$$
\sigma=\frac{(\partial g)}{\partial A} \mathrm{p}, \mathrm{T}, \mathrm{ni}(3)
$$

En el caso de que una solución acuosa que contiene partículas que pueden ser adsorbidas, se encuentre en equilibrio con una superficie adsorbente, las ecuaciones de Gibbs-Duhem para el área de la superficie (4) y para la solución libre (5) se expresan de la manera siguiente:

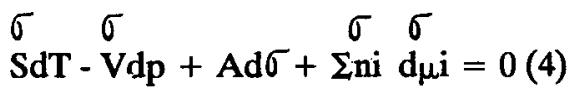


SdT - Vdp $+\sum_{i} n i d \mu i=0(5)$

En equilibrio $\sigma_{\mu} \dot{i}=\mu \mathrm{i}$, de modo que para presión y temperatura constantes $(d T=d p=0)$ se reduce el sistema de ecuaciones a:

$$
-A d \sigma=\underset{i}{\Sigma}(\mathrm{Ni}-\mathrm{Ni}) \mathrm{d} \mu \mathrm{i}(6)
$$

Para el potencial químico $y$ i rige:

$$
\mu \mathrm{i}=\mu \mathrm{io}+\mathrm{RT} \ln \left(\mathrm{Ci} \gamma_{\mathrm{i}}\right)
$$

Donde $\mathrm{R}$ es una constante, $\mathrm{Ci}$ la concentración del componente i; $\gamma \mathrm{i}$ el coeficiente de actividad de i y $\mu$ io el potencial químico inicial.

Así la ecuación (6) se convierte en:

$$
-\operatorname{Ad} \sigma=\operatorname{RT} \Sigma\left(\stackrel{\sigma}{(N i-N i)} \operatorname{dln}\left(\mathbf{C i} \gamma_{i}\right)\right.
$$

En caso de que la tensión superficial descienda con un aumento de la actividad (d $\sigma / \mathrm{dl}(\mathrm{ciri})<0)$; así $(\mathrm{Ni}-\mathrm{Ni})$ se hace $>0$; la sustancia disuelta pasa a la superficie de ruptura de fases (adsorción positiva) (7). En caso contrario se empobrece la superficie en cantidad de sustancia disuelta (adsorción negativa).

La diferencia ( $\mathrm{Ni}$ - Ni)representa la cantidad del componente i adsorbida en la superficie del adsórbente. Las variables A y Ni pueden ser referidas a la masa del adsorbente de la manera siguiente:

$$
\text { F. } \underset{M_{A}}{A}=S A ; \underset{M A}{\stackrel{\sigma}{N i}-\mathrm{Ni}}=A i(9)
$$

Los cambios de la tensión superficial entre el valor original $\sigma_{0}$ al valor en equilibrio rse expresan a través de la presión bidimensional de la capa de adsorción $\pi$ :

$$
\sigma_{0}-\sigma=\pi(10)
$$

La combinación de las ecuaciones (8) hasta (10) bajo la condición de una conducta ideal de la solución acuosa $(i=1)$ conduce a la expresión

$$
\mathrm{S}_{\mathrm{A}} \mathrm{d} \pi=\mathrm{RT}_{\mathbf{i}} \text { aidln } \mathrm{Ci}=0 \text { (11) }
$$

Las ecuaciones (6); (8) y 11) representan formas diferentes de la isoterma de adsorción de Gibbs. 


\section{Isotermas de adsorción}

Las isotermas de adsorción son expresiones matemáticas, que derivadas de la ecuación de Gibbs describen la relación entre la concentración en equilibrio del soluto y la cantidad adsorbida. Una de las más conocidas es la de Langmuir que puede ser formulada de la manera siguiente:

$$
A=A \max \quad \begin{gathered}
\beta C e q \\
1+\beta C \text { eq }
\end{gathered}
$$

Donde a es la cantidad adsorbida; Amax es la máxima cantidad adsorbida; $\beta$ una constante y Ceq la concentración de la solución en equilibrio.

Mediante transformación matemática esta ecuación puede expresarse de esta manera:

$$
\frac{1}{a}=\frac{1}{A \max }+\frac{1}{A \max \beta} \cdot \frac{1}{\mathrm{Ceq}}
$$

Si se grafica $\frac{1}{\mathrm{a}}$ Vs. $\frac{1}{\mathrm{Ceq}}$ se obtiene una línea recta en la cual $\frac{1}{\mathrm{Amax}}$ es el punto de intersección con el eje de las ordenadas y $\frac{1}{A_{\max }} \beta$ es la pendiente.

La ecuación de Langmuir mostró ser una buena relación matemática de la adsorción, pero es sólo aplicable a los procesos de equilibrio reversibles y no puede aplicarse en la descripción de los procesos de quimioadsorción con la formación de enlaces fuertes, así como tampoco a superficies heterogéneas como el carbón activado, pues asume que todos los puntos de la superficie del adsorbente tienen igual nivel energético, lo cual no es cierto en el caso de las citadas superficies heterogéneas.

La heterogeneidad energética de la superficie del sólido está relacionada con la aplicabilidad de la ecuación de Freundlich que se expresa como sigue:

$$
A=K_{\text {eq }}^{\mathbf{n}}
$$

Donde K y n son constantes.

De la logaritmización de esta ecuación resulta:

$$
\ln =\operatorname{Ln} K+n \ln C_{e q}
$$

Que como se observará es la ecuación de una línea recta, en la que lnK es el punto de insercción de las coordenadas; $n$ es la pendiente de la recta.

Numerosas isotermas han sido planteadas para explicar la conducta de adsorción. Las hay incluso de tres parámetros, aunque las más utilizadas y sencillas son las de dos parámetros como las de Langmuir y Freundlich. 


\section{Cinética de adsorción}

El proceso de adsorción de una sustancia en solución acuosa en la superficie de un cuerpo sólido se puede subdividir en cuatro pasos fundamentales:

Difusión de la sustancia disuelta en la fase líquida.

Difusión de la partícula del soluto a.través de la capa de ruptura de fase líquida-superfice externa del cuerpo sólido (difusión fímica).

Difusión de la partícula de soluto en el interior del grano (prodifusión, difusión superficial)

Interacción de adsorción en el interior de la superficie del adsorbente.

Los pasos 1 y 4 pueden ser desechados en virtud de que transcurren relativamente rápido y por consiguiente no determinan la velocidad.

La expresión cinética para la difusión fílmica se expresa:

$$
\frac{-d c}{d t}=K_{f} a_{v}\left(C-C_{s}\right)
$$

Donde $\mathbf{C}$ es la concentración del soluto en la fase líquida; Cs la concentración del soluto en las cercanías del grano; av superficie relacionada al volumen; $K_{f}$ coeficiente del transporte de materia para la difusión fílmica; $t$ tiempo.

En el caso de la difusión en el interior del grano, se puede diferenciar entre una difusión en los poros llenos de solución acuosa (porodifusión superficial). El paso de la porodifusión de los poros (difusión superficial). El paso de la porodifusión se describe mediante la siguiente expresión, derivada de la segunda ley de Fick.

$$
\frac{\partial \mathrm{C}}{\partial \mathrm{t}}=\frac{\mathrm{D}_{\mathrm{p}}\left(\partial^{2} \mathrm{C}\right.}{\partial \mathrm{r}^{2}}+\frac{2}{\mathrm{r}} \frac{\partial \mathrm{C})}{\partial \mathrm{r}}
$$
el grano.

Donde Dp es el coeficiente de la porodifusión; r es la coordenada local en

Para el caso de la difusión superficial, la segunda ley de Fick puede ser escrita de la manera siguiente:

$$
\frac{\partial \mathrm{a}}{\partial \mathrm{t}}=\mathrm{D}_{\mathrm{s}} \frac{\left(\partial^{2} \underline{\mathrm{s}}+2\right.}{\partial \mathrm{r}^{2}}-\frac{\partial \mathrm{a})}{\mathrm{r}} \frac{\mathrm{g}}{\partial \mathrm{r}}
$$

En la que Ds es el coeficiente de la difusión superficial. La ecuación anterior puede ser expresada más fácilmente mediante la fórmula propuesta por Glückauf (8):

$$
\frac{\partial \mathrm{a}}{\partial \mathrm{t}}=\mathrm{K}_{\mathrm{s}} \mathrm{a}_{\mathrm{v}} \boldsymbol{\beta}\left(\mathrm{a}_{\mathrm{s}}-\overline{\mathrm{a}}\right)
$$


En la que a es la cantidad media adsorbida en el adsorbente; as cantidad adsorbida en la cercanía del grano; $\mathrm{K}_{\mathbf{s}}$ coeficiente de transporte de materia; $\beta$ factor de corrección.

Entre los cocficientes de transporte de materia y de difusión existe la siguiente relación:

$$
\begin{array}{r}
K_{s} a_{v}=15 D_{s} \\
R^{2}
\end{array}
$$

Donde $\mathbf{R}$ es el radio de la partícula adsorbente.

Para la descripción matemática de la cinética de adsorción, se hace necesario combinar las ecuaciones de transporte, con ecuaciones de isortermas y de balance de materias.

Los diferentes modelos descritos en la literatura, se diferencian en la asunción del mecanismo de transporte dominante y de la isoterma de adsorción que rige.

A menudo pueden describirse los equilibrios de adsorción a trâvés de la ecuación de Freundlich. Ejemplo de esos casos es el modelo de Hand, Crittenden y Thacher (9) para el cálculo de coeficientes de difusión asumiendo una isoterma de Freundlich para una difusión superficial como mecanismo de transporte dominante. Suzuki y Kawazoe encontraron soluciones para la porodifusión (10) así como para la difusión superficial (11). El modelo propuesto por Pell, Benedek y Crowe (12) contempla tanto la difusión fílmica como la difusión interna, esta última subdividida en macroporodifusión y microporodifusión.

Una solución analítica para isotermas lineares y difusión superficial dominante fue propuesta por Aguwa, Patterson, Haas y Noll (13) basados en el modelo de difusión de Crank (14).

Otros modelos cinéticos han servido para estudiar la influencia de las condiciones hidrodinámicas como el flujo, la velocidad lineal, etc. Entre ellos se encuentran trabajos realizados por Berninger y Vortmeyer (15). Van Deventer (17) propuso un modelo para la adsorción reversible de cianuro de oro en carbón activado.

Recientemente ha sido usada la técnica de la espectroscopia de pérdida de energía del electrón, para estudios de cinética de adsorción y desorción, entre los que sobresalen los de Dubois, Ellis, Zegaski y Kevan $(17,18)$.

\section{Dinámica de adsorción}

El tratamiento de los procesos de adsorción en una columna de adsorbentes de largo $h$ y porosidad $b$, que es atravesada por un líquido con una velocidad $\mathrm{V}$, parte del siguiente balance de masas: 


$$
\frac{\varepsilon b}{\partial t}+\frac{V \partial c}{\partial n} \frac{\partial a}{\partial t} \rho_{b}=0
$$

donde $\rho_{b}$ es la densidad de empacamiento de la columna.

Esta ecuación presenta los cambios en el tiempo y el espacio de la concentración del soluto, así como los cambios en el tiempo de la cantidad adsorbida. La representación gráfica de la concentración reducida $\mathrm{C}=(\mathrm{C} / \mathrm{Co})$ contra el tiempo $(t)$ arroja la curva de lecho fijo del adsorbente.

La toma de la curva de lecho fijo tiene una gran importancia para la colocación de filtros en la limpieza de aire en el tratamiento del agua. De una curva de lecho fijo se pueden obtener importantes informaciones como la cantidad adsorbida a determinada altura de la columna a un tiempo $(\mathrm{t})$; así como la proporción entre carga útil (cantidad adsorbida al momento de la ruptura) y la cantidad adsorbida en equilibrio; las condiciones óptimas de experimento para el más adecuado uso de la capacidad de adsorción, etc.

En una curva de lecho fijo se forman normalmente tres zonas:

Zona de equilibrio: En la cercanía de la entrada del líquido en el adsorbente, se ha alcanzado la cantidad adsorbida de equilibrio; no se adsorbe más y la concentración Co del soluto permanece constante y está en equilibrio con la cantidad adsorbida Ao.

Zona de adsorción: En esa zona se desarrolla la adsorción. La concentración del soluto desciende de Co a cero y la cantidad adsorbida dentro de la zona aumenta hasta el valor de saturación. El tamaño de la zona de adsorción está determinada esencialmente por la velocidad de adsorción.

Zona libre de soluto: La tercera zona se forma entre la zona de adsorción y la salida de la columna. Aquí hay prácticamente adsorbente no cargado de soluto.

Para la descripción de curvas de lecho fijo son necesarios varios parámetros, los cuales son definidos según el modelo usado. En general son parámetros que caracterizan las condiciones de equilibrio, la altura de la columna, el tiempo de contacto y el transporte de masa. Hay dos tiempos que deben ser observados de manera particular; son el tiempo de ruptura (o tiempo de retención) y el tiempo de saturación.

\section{Mecanismos de transporte en la dinámica de adsorción}

Tal como vimos en la cinética de adsorción, es en la dinámica también importante si el mecanismo dominante de transporte es la difusión superficial o la porodifusión, o la combinación de ambas. Algunos modelos toman en consideración también la difusión fílmica. Por lo regular, los efectos axiales de dispersión son descartados, a pesar de que algunos autores los toman en cuenta $(19,20,21)$. 
Numerosos modelos han sido propuestos para la descripción de la dinámica de adsorción, los cuales asumen determinadas isotermas y mecanismos de transporte. La mayoría toma por lo regular o una isoterma de Langmuir o de Freundlich.

Hay modelos que se han desarrollado para describir la dinámica de adsorción de una sola sustancia, mientras otros describen la de mezclas de sustancias.

Entre los primeros se destacan los de Mohtadi y Krieger (22) y los de Hashimoto y Miura $(23,24)$, entre los segundos pueden citarse los de Famularo, Müller y Pannu (25), el de Wang y Tien (26) y los de Merk, Fritz y Schlünder (27).

Principios cinéticos para la descripción de curvas de lecho fijo.

Para la difusión filmica, la solución de Glückauf plantea la siguiente ecuación:

$$
\frac{\mathrm{da}}{\mathrm{dt}}=\frac{\mathrm{K}_{\mathrm{fav}}}{\rho_{\mathrm{b}}}\left(\mathrm{C}-\mathrm{C}_{\mathrm{s}}\right)
$$

Mientras que para la difusión superficial:

$$
\frac{\mathrm{da}}{\mathrm{dt}}=\mathrm{K}_{\mathrm{s}} \mathrm{av}_{\mathrm{v}} \beta\left(\mathrm{a}_{\mathrm{s}}-\mathrm{a}\right)
$$

Donde $\mathrm{K}_{f a v}$ y $\mathrm{K}_{\mathrm{s}} \mathrm{av}_{\mathrm{v}}$ respectivamente son los coeficientes de transporte de materia de ambos procesos.

\section{Conclusión}

Los fenómenos de adsorción, estudiados en sus tres aspectos principales: equilibrio, cinética y dinámica, constituyen un importante instrumento en la lucha por la preservación del medio ambiente. El conocimiento de la conducta de adsorción de una sustancia o de mezclas de ellas, tiene una vital importancia para la preservación de la limpieza del aire y el tratamiento de las aguas.

\section{BIBLIOGRAFIA}

Romankov, P.G.; N. B. Raskovskaja; V. F. Faolou. Proceso de transporte de materia de la tecnología química. Edición alemana. Adkademie - Verlag. Berlín, 1979.

Langmuir, I. J. American Chem. Soc. 38 (1916) 221.

Langmuir, I. J. American Chem. Soc. 40 (1918) 1361

Phelps, H. J.; R. A. Peters. Proc. Royal Soc. 124 (1929) 554. 
Snoeyink, V. L.; W. J. Weber Jr.; H. B. Mark. J. Environ. Sci. Techn. 3 (1969) 918

Schilow, N.; K. Tschmuton. Z. Phys. Chem. 28 (1924) 992.

Schwabe, K. Physikalische Chemie. Akademie Verlag, Berlín 1973.

Glückauf, E. Trans. Faraday Soc. 51 (1953) 1540.

Hand, D. W.; J. C. Crittenden; W. E. Tahcker. J. Environ. Engng. 109 (1983) 82.

Suzuki, M.; K. Kawazoe. J. Chem. Engn. Japan 7 (1974) 275.

Peel, R. G.; A. Benedek; C. M. Crowe. AICHE Journal 27 (1981) 25

Agwa, A. A.; J. W. Patterson; C. N. Haas; K. E. Noll. Journal WPCF 56 (1984) 442.

Crank, J. J. Mathematics of diffusion. Clarendon Press, Oxford, 1956.

Berninger, R.; D. Vortmeyer. Chem Ing. Tech. 59 No. 3 (1987) 224.

Van Deventer, J. S. J. Chem. Eng. Commun. 44 (1986) 257.

Dubois, L. H.; T. H. Ellis; B. R. Zegarski; S. D. Kevan Surface Science 172 (1986) 385.

Dubois, L. H.; T. H. Ellis; S. D. Kevan. Journal of electron spectroscopy and related phenomena 39 (19867) 27.

Radcliffe, D. F. Chem. Engn. Commun. 25 (1984) 183.

Vermeulen, T.; G. Klein; N. K. Hiester. Adsortion and Ion exchange. Chemical Engineers Handbook. Ed. McGraw-Hill, New York 1973.

Charkravorti, R. K.; T. W. Weber. AICHE Symposium Series 71 (1975) 392.

Mohtadi, M.; U. Krieger. GWF - WASSER/Abwasser 122 (1981) 513.

Hashimoto, K.; K. Miura. J. Chem. Engn. Japan 9 (1976) 388.

Miura, K. : K. Hashimoto. J. Chem. Engn. Japan 10 (1977) 490.

Famularo, J.; J. A. Müller; A. S. Pannu. Journal WPCF 52 (1980) 2019.

Wang, S.; C. Tien. AICHE Journal 28 (182) 565.

Merk, W.; W. Fritz; E. U. Schlünder. Chem. Engn. Sci. 36 (1981) 743. 